\title{
Multivariable Integral Control of Resonant Structures
}

\author{
B. Bhikkaji, S. O. R. Moheimani and I. R. Petersen
}

\begin{abstract}
Integral resonant control (IRC) is a feedback control technique used for damping active structures with a collocated sensor/actuator pair. This paper extends this control technique to structures having several collocated sensor/actuator pairs. Conditions for the closed loop stability are derived, and the set of such stabilizing IRC controllers is shown to be a convex set. An experimental implementation of an IRC controller on an active structure (Cantilever beam) with two pairs of bonded collocated piezoelectric sensors/actuators is also presented.
\end{abstract}

\section{INTRODUCTION}

Flexible structures are resonant systems prone to high amplitude mechanical vibrations. Many industrial, aerospace and scientific applications incorporate flexible structures, see [1] and [2]. As they are highly resonant, even small disturbances can trigger high amplitude vibrations. Most flexible structures are distributed parameter systems with their dynamics being governed by partial differential equations. Therefore, in theory, they posses an infinite number of lightly damped resonant modes. In other words their transfer-functions are of infinite order with poles close to the imaginary axis, making them very difficult to control.

In a practical scenario, the PDE model is discretized to form a lumped model or alternatively the infinite order transfer-function is truncated and only the first few modes are retained. Using the truncated model, feedback controllers are designed to damp the resonant modes of the structure. The truncated modes or the discarded modes are referred to as the out of bandwidth modes and play no part in the controller design. The presence of the uncontrolled truncated modes can lead to what is known as the spillover effect, [3]. That is, the control energy is channeled to the residual modes which may destabilize the closed loop system.

In order to overcome the spillover effect, collocated sensors and actuators have been used, and feedback controllers linking these sensors and actuators, that guarantee unconditional closed loop stability have been investigated. Velocity feedback, [4] and [5] and Resonant controllers, [6] are two well known examples of controllers that guarantee unconditional closed loop stability of the system. Positive position feedback (PPF), [7] and [8], is another technique which is insensitive to the spill over effect and have similar stability properties. In [10] the authors had shown

Dr. B. Bhikkaji and Prof. S. O. R. Mohiemani are with the School of Electrical Engineering and Computer Science, University of Newcastle, Callaghan, NSW, 2308, Australia, Bharath.Bhikkaji@newcastle.edu.au, Reza.

Moheimanianewcastle.edu.au

Prof. I. R. Petersen with the School of Information Technology and Electrical Engineering, University of NSW, Australian Defence Force Academy campus,Canberra, Australia, irp@ee.adfa.edu.au the conditional closed loop stability of PPF on collocated structures. Unlike velocity feedback and resonant control, the frequency response of a PPF controller rolls off quickly at high frequencies, thus leaving the unmodeled high frequency modes of the plant undisturbed.

In [9], the authors proposed a control technique known as Integral resonant control (IRC). This technique was proposed for a flexible structure with a single sensor/actuator pair (i.e., for the SISO case). It was observed in [9] that IRC resulted in good damping and also the controller rolled off quickly at high frequencies. This paper extends the work of [9] to the case of multiple collocated sensor/actuator pairs (i.e., MIMO systems). Stability conditions for MIMO IRC are derived and the possibility of making them unconditionally stable is also discussed. Moreover due to its parametric structure, the MIMO IRC, like the SISO case, rolls off quickly at the high frequencies.

\section{Problem Statement}

Flexible structures with collocated sensor/actuator pairs posses a model structure of the following form (see [8] and [11]):

$$
\begin{aligned}
\ddot{X}+M \dot{X}+\Omega X & =\Psi u \\
y & =\Psi^{\top} X+D_{u} u
\end{aligned}
$$

where $X$ is an $n \times 1$ vector, $M$ and $\Omega$ are $n \times n$ positive definite diagonal matrices, $\Psi$ is an $n \times m$ matrix, $u$ is an $m \times 1$ vector of inputs and $D_{u}$ is an $m \times m$ matrix. It is worth noting that both $y$ and $u$ are of the same dimension $m$ as the sensors (outputs) and actuators (inputs) appear in pairs. In summary (1) represents a system with $m$ collocated sensoractuator pairs, and describing the dynamics of the system up to the $n^{\text {th }}$ mode. The goal of this paper is to construct multivariable controllers for resonant structures of the form (1).

In [9] the authors considered a special case of (1) with $\Psi$ being an $m \times 1$ vector, the input $u$ being a scalar function and $D_{u}$ being replaced by the scalar $d_{u}$. In the Laplace domain the transfer-function of this SISO system can be written as

$$
G_{n}(s)=\sum_{k=1}^{n} \frac{\psi_{k}^{2}}{s^{2}+2 \sigma_{k} \omega_{k} s+\omega_{k}^{2}}+d_{u},
$$

where $\Psi=\left[\psi_{1}, \psi_{2}, \ldots, \psi_{n}\right]^{\top}, \quad M=$ $\operatorname{diag}\left(2 \sigma_{1} \omega_{1}, 2 \sigma_{2} \omega_{2}, \ldots, 2 \sigma_{n} \omega_{n}\right)$ and $\Omega \quad=$ $\operatorname{diag}\left(\omega_{1}^{2}, \omega_{2}^{2}, \ldots, \omega_{n}^{2}\right)$. The approach of [9] achieved significant damping by adding a feed through term $d$ and wrapping an integral controller $C(s)=\frac{\gamma}{s}$ around it; see 
Figure 1. In [9], to analyze the stability of the closed loop system, $G_{n}(s)$, (2), was approximated by

$$
\hat{G}_{n}(s)=\sum_{k=1}^{n} \frac{\psi_{k}^{2}}{s^{2}+\omega_{k}^{2}}+d_{u} .
$$

It was shown that the poles and zeros of $\hat{G}_{n}(s)$, (3), were purely imaginary and their Pole-Zero map (PZ map) had a pole-zero alternating pattern as shown in Figure 2(a). Adding a suitable $d$ term to $\hat{G}_{n}(s)$ shifted the zeros in such a way that $\hat{G}_{n}(s)+d$ had a PZ map with a zero-pole alternating pattern, see Figure 2(a). It was found that the root-locus plot of $\frac{1}{s}\left(\hat{G}_{n}(s)+d\right)$ lied entirely in the left half plane. Therefore by choosing a suitable gain $\gamma>0$, a significant amount of damping could be achieved; see Figure 3. Note that this amounts to wrapping $C(s)=\frac{\gamma}{s}$ around $\hat{G}_{n}(s)+d$.

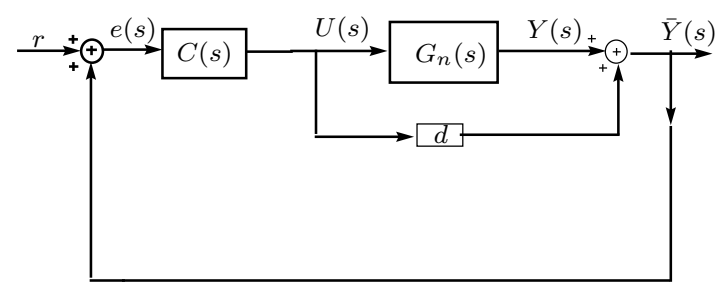

Fig. 1. Closed-loop system with the Integral controller $C(s)=\frac{\Gamma}{s}$ around $G_{n}(s)+d$. (a)

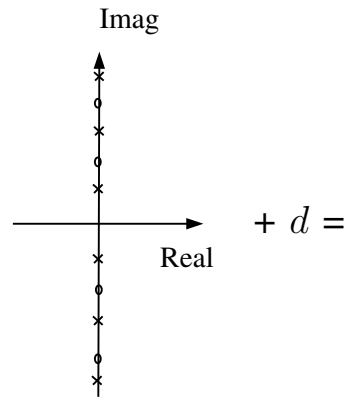

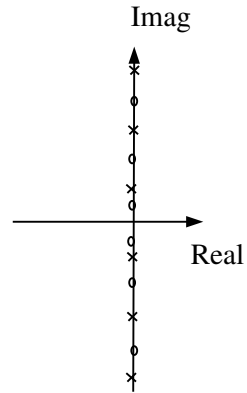

(b)
Fig. 2. Illustration of the Pole-Zero positions of $\hat{G}_{n}(s)$, (3), and $G_{n}(s)+d$, ' $\mathrm{x}$ ' denoting poles and 'o' denoting zeros.

In this paper, the IRC scheme is extended to the MIMO case; i.e., the general case presented in (1). A natural extension of the above mentioned scheme to the MIMO case is to replace the scalar feed through term $d$ by an $m \times m$ matrix $D$, and the scalar gain $\gamma$ to an $m \times m$ matrix $\Gamma$. However, unlike the SISO case, proving the closed loop stability needs more advanced mathematical machinery than standard root-locus techniques.

Let

$$
G_{n}(s) \triangleq \Psi\left[s^{2} I+M s+\Omega\right]^{-1} \Psi^{\top}
$$

denote the transfer-function corresponding to the MIMO system (1), $C(s)$ denote the MIMO integral controller $C(s)=\frac{\Gamma}{s}$

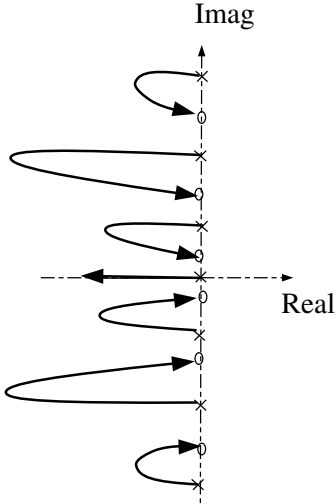

Fig. 3. Illustration of the root locus of $\frac{1}{s}\left(\hat{G}_{n}(s)+d\right)$.

with $\Gamma$ being an $m \times m$ matrix, and $D$ denote the $m \times m$ feed-through matrix. Before proving the closed-loop stability, for technical ease, the above mentioned control scheme is rewritten into an equivalent form. This equivalent form makes it easier to analyze the stability.

Note, from Figure 1 that the input to the controller $C(s)$ is given by

$$
e(s)=r(s)+\tilde{Y}(s)
$$

where

$$
\tilde{Y}(s)=Y(s)+D U(s),
$$

with $U(s)$ and $Y(s)$, (1), being the plant inputs and outputs respectively. The output of the controller, which is the plant input $U(s)$, is given by $U(s)=\frac{\Gamma}{s} e(s)$. Therefore,

$$
U(s)=\frac{\Gamma}{s} r(s)+\frac{\Gamma}{s} Y(s)+\frac{\Gamma}{s} D U(s),
$$

which implies

$$
[s I-\Gamma D] U(s)=\Gamma r(s)+\Gamma Y(s),
$$

and hence

$$
\begin{aligned}
U(s)= & {[s I-\Gamma D]^{-1} \Gamma r(s) } \\
& +[s I-\Gamma D]^{-1} \Gamma Y(s) .
\end{aligned}
$$

As $Y(s)=G_{n}(s) U(s)$, it can be further deduced that

$$
\begin{aligned}
Y(s)= & G_{n}(s)[s I-\Gamma D]^{-1} \Gamma r(s) \\
& +G_{n}(s)[s I-\Gamma D]^{-1} \Gamma Y(s),
\end{aligned}
$$

which implies

$$
\begin{aligned}
& {\left[I-G_{n}(s)[s I-\Gamma D]^{-1} \Gamma\right] Y(s)=} \\
& G_{n}(s)[s I-\Gamma D]^{-1} \Gamma r(s) .
\end{aligned}
$$

It can be inferred from equation (11) that the control strategy presented in Figure 1 is equivalent to the one presented in Figure 4, with

$$
K(s)=[s I-\Gamma D]^{-1} \Gamma .
$$

Therefore proving the stability of the control strategy presented in Figure 1 is equivalent to proving the stability of the control strategy presented in Figure 4. 


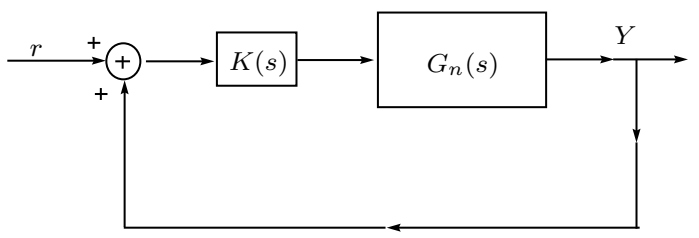

Fig. 4. Closed-loop system with the controller $K(s)$ around $G_{n}(s)$.

\section{A. Proof of closed loop stability}

The proof presented here for the internal stability of the feedback system with the controller $K(s)$ uses the mathematical machinery provided by [10].

Let

$$
\begin{aligned}
\mathcal{C}_{1} \triangleq & \left\{R(s) \in \mathcal{R H}_{\infty}^{m \times m}:\right. \\
& \left.\mathrm{j}\left[R(\mathrm{j} \omega)-\mathrm{R}^{*}(\mathrm{j} \omega)\right] \geq 0, \forall \omega \in(0, \infty)\right\}
\end{aligned}
$$

and

$$
\begin{aligned}
\mathcal{C}_{2} \triangleq & \left\{R(s) \in \mathcal{R H}_{\infty}^{m \times m}: R(\infty) \geq 0,\right. \\
& \left.\mathrm{j}\left[R(\mathrm{j} \omega)-\mathrm{R}^{*}(\mathrm{j} \omega)\right]>0, \forall \omega \in(0, \infty)\right\},
\end{aligned}
$$

where $\mathcal{R} \mathcal{H}_{\infty}^{m \times m}$ denotes the set of all $m \times m$ matrices of stable rational transfer-functions that are uniformly bounded on the imaginary axis, [12].

It can be shown that, if $P(s) \in \mathcal{C}_{1}$ and $Q(s) \in \mathcal{C}_{2}$ are such that $P(\infty) Q(\infty)=0$, then a positive feedback connection of $P(s)$ and $Q(s)$ is internally stable iff $\lambda_{\max }(P(0) Q(0))<1$. Formally stated

Theorem: Given $P(s) \in \mathcal{C}_{1}$ and $Q(s) \in \mathcal{C}_{2}$ such that $P(\infty) Q(\infty)=0$, then their positive feedback interconnection is internally stable if and only if the eigenvalues of the matrix $P(0) Q(0)$ are strictly less than one.

Proof: Refer to [10].

It can be inferred directly from (4) and (12) that $G_{n}(s) \in$ $\mathcal{C}_{1}$ and $K(\infty)=0$ respectively. Therefore, for $K(s)$ to be in $\mathcal{C}_{2}, K(s)$ must be stable and $K(\mathrm{j} \omega)$ must satisfy

$$
\mathrm{j}\left[K(\mathrm{j} \omega)-K^{*}(\mathrm{j} \omega)\right]>0 .
$$

In the following it will be shown that for $\Gamma$ and $D$ both symmetric, with $\Gamma$ being positive definite and $D$ being negative definite (or $-D$ being positive definite), $K(s)$ would belong to the set $\mathcal{C}_{2}$.

Note that as the product $-\Gamma D$ is strictly positive definite, stability of $K(s)$, is direct from (12). As $\Gamma$ and $-D^{-1}$ are symmetric and positive definite there exists a non-singular $n \times n$ matrix $X$ such that

$$
X^{\top} D^{-1} X=\Lambda
$$

and

$$
X^{\top} \Gamma X=\mathrm{I},
$$

where $\Lambda$ is a diagonal matrix and $I$ is the identity matrix. The elements of $\Lambda$ are eigenvalues of $\Gamma^{-1} D^{-1}$, see [13]. As $\Gamma$ and $-D$ are positive definite, every eigenvalue of the product $\Gamma D$, and hence $(\Gamma D)^{-1}$, is negative, see [14]. Therefore, $\Lambda=\operatorname{diag}\left(-\lambda_{11},-\lambda_{22}, \ldots,-\lambda_{n n}\right)$, for some $\lambda_{k k}$ ' strictly positive.

Note that $\Gamma=X^{-\top} X^{-1}$ and $D^{-1}=X^{-\top} \Lambda X^{-1}$, which implies $D=X \Lambda^{-1} X^{\top}$ and $\Gamma D=X^{-\top} \Lambda^{-1} X^{\top}$. Hence

$$
\begin{aligned}
K(s) & =[s I-\Gamma D]^{-1} \Gamma \\
& =\left[s I-X^{-\top} \Lambda^{-1} X^{\top}\right]^{-1} X^{-\top} X^{-1} \\
& =X^{-\top}\left[s I-\Lambda^{-1}\right]^{-1} X^{-1},
\end{aligned}
$$

This in turn implies

$$
K(\mathrm{j} \omega)=X^{-\top}\left[\mathrm{j} \omega \mathrm{I}-\Lambda^{-1}\right]^{-1} X^{-1}
$$

and

$$
K^{*}(\mathrm{j} \omega)=X^{-\top}\left[-\mathrm{j} \omega \mathrm{I}-\Lambda^{-1}\right]^{-1} X^{-1},
$$

and hence

$$
\begin{aligned}
& \mathrm{j}\left[K(\mathrm{j} \omega)-\mathrm{K}^{*}(\mathrm{j} \omega)\right]=\mathrm{j} X^{-\top}\left(\left[\mathrm{j} \omega \mathrm{I}-\Lambda^{-1}\right]^{-1}\right. \\
& \left.-\left[-\mathrm{j} \omega \mathrm{I}-\Lambda^{-1}\right]^{-1}\right) X^{-1} .
\end{aligned}
$$

Setting $\alpha_{k}=\frac{1}{\lambda_{k k}}, k=1,2, \ldots, n$, it can be seen that

$$
\begin{aligned}
& {\left[\mathrm{j} \omega \mathrm{I}-\Lambda^{-1}\right]^{-1}} \\
& =\left[\begin{array}{cccc}
\frac{1}{j \omega+\alpha_{1}} & 0 & \cdots & 0 \\
0 & \frac{1}{j \omega+\alpha_{2}} & \cdots & 0 \\
0 & 0 & \ddots & 0 \\
0 & 0 & \cdots & \frac{1}{j \omega+\alpha_{n}}
\end{array}\right] \\
& =\left[\begin{array}{cccc}
\frac{-\mathrm{j} \omega+\alpha_{1}}{\omega^{2}+\alpha_{1}^{2}} & 0 & \ldots & 0 \\
0 & \frac{-\mathrm{j} \omega+\alpha_{2}}{\omega^{2}+\alpha_{2}^{2}} & \ldots & 0 \\
0 & 0 & \ddots & 0 \\
0 & 0 & \ldots & \frac{-\mathrm{j} \omega+\alpha_{n}}{\omega^{2}+\alpha_{n}^{2}}
\end{array}\right]
\end{aligned}
$$

It is evident from (21) and (22) that $\mathrm{j}\left[K(\mathrm{j} \omega)-\mathrm{K}^{*}(\mathrm{j} \omega)\right]$ is strictly positive definite. Thus for $\Gamma$ and $-D$ positive definite $K(s) \in \mathcal{C}_{2}$. Furthermore, for the closed loop to be stable $\lambda_{\max }\left(G_{n}(0) K(0)\right)<1$ has to hold. As $G_{n}(0)=\Psi^{\top} \Omega^{-1} \Psi$ and $K(0)=-D^{-1}$, this implies

$$
-D>\Psi^{\top} \Omega^{-1} \Psi .
$$

Remark 1: If the controllers $K_{1}(s)=\left[s I-\Gamma_{1} D_{1}\right]^{-1} \Gamma_{1}$ and $K_{2}(s)=\left[s I-\Gamma_{2} D_{2}\right]^{-1} \Gamma_{2}$ satisfy (15), then so does $K^{(2)}(s)=K_{1}(s)+K_{2}(s)$. Infact for any finite sum $K^{(n)}(s)=\sum_{k=1}^{n} K_{k}(s)$. with each $K_{k}(s)=$ $\left[s I-\Gamma_{k} D_{k}\right]^{-1} \Gamma_{k}$ satisfying $(15), K^{(n)}(s)$ would also satisfy (15). For $K^{(n)}(s)$ to provide closed loop stability $\Gamma_{k}>0, D_{k}<0, k=1,2,3, \ldots, n$ and $-\sum_{k=1}^{n} D_{k}^{-1}>$ $\Psi^{\top} \Omega^{-1} \Psi$. These constraints can also be posed as LMIs.

Remark 2: The constraint (23) complements the constraint that $D$ has to be negative definite. Moreover, if $g_{0}=$ $\sup _{n} G_{n}(0)$ is known, then choosing $-D>g_{0}$ would give stability over any finite number of out of bandwidth modes. In any physical system, power and bandwidth constraints 


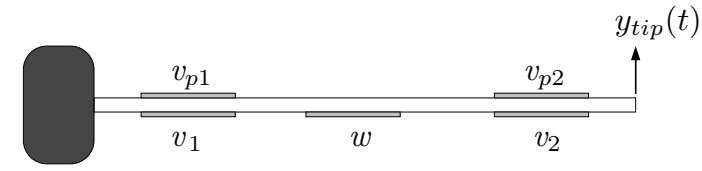

Fig. 5. Layout of the cantilever beam, $v_{1}$ and $v_{2}$ denote the actuator patches, $v_{p 1}$ and $v_{p 2}$ denote the sensor patches, $w$ denotes the noise patch and $y_{\text {tip }}(t)$ denotes tip displacement.

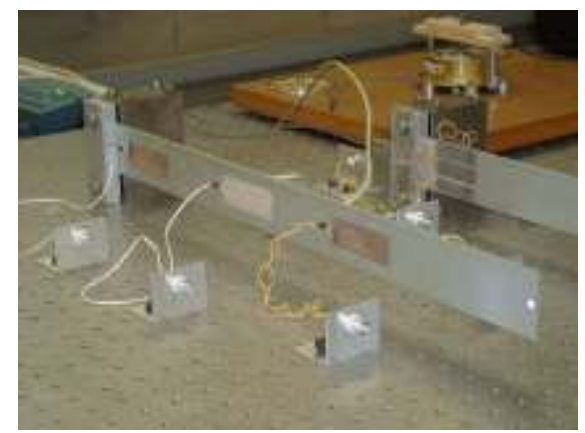

Fig. 6. Picture of the cantilever beam

ensure that it is not possible to excite all of the infinite modes of the system. Hence, for $-D>g_{0} \mathrm{I}$ and $\Gamma>0$, the IRC controller will guarantee closed loop stability for all practical purposes.

\section{EXPERIMENTAL SETUP}

Here, a Cantilever beam representing a physical resonant system is considered. This beam, which is clamped at one end and free at the other end, is susceptible to high amplitude vibrations when disturbed. In this section, a controller of the form (12) will be designed to damp these highly resonant vibration modes of the beam.

As mentioned above, the cantilever beam is clamped at one end and free at the other. Two pairs of piezoelectric patches are attached to this beam, one pair located close to the clamped end and the other pair located close to the free end of the beam. For each pair, one piezoelectric patch will be used as an actuator (where input signals are applied) and the other patch will act as a sensor (where output signals are recorded). Another solitary piezoelectric patch is attached to the center of the beam and will be driven by a voltage source $w$. This voltage $w$ represents the disturbance on the beam. See Figures 5 and 6 for a schematic beam set up and an actual picture of the beam respectively.

\section{A. System identification}

The experimental setup is treated as a three-input-threeoutput multivariable system, see Figure 7 . The inputs ( $v_{1}$ and $v_{2}$ ) in Figure 7 are the voltages applied to the actuators of the collocated piezoelectric patches and the outputs $v_{p 1}$ and $v_{p 2}$ are the voltages induced at the corresponding sensors. The third input $w$ is the disturbance on the beam and the output $y_{\text {tip }}$ is the displacement of the tip of the beam.

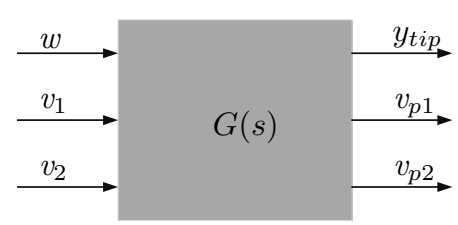

Fig. 7. Augmented MIMO plant

Since the system is modeled as a three-input-three-output system, the frequency response function (FRF) $G(\mathrm{j} \omega)$ is a $3 \times 3$ matrix with each element $G_{i j}(\mathrm{j} \omega), i, j=1,2$ and 3 , corresponding to a particular combination of the input and the output i.e., $G_{i j}(\mathrm{j} \omega)=G_{y_{i} u_{j}}=Y_{i}(\mathrm{j} \omega) / U_{j}(\mathrm{j} \omega)$, where $y_{1}=y_{\text {tip }}, y_{2}=v_{p 1}$ and $y_{3}=v_{p 2}$, and $u_{1}=$ $w, u_{2}=v_{1}$ and $u_{3}=v_{2} . Y_{i}(\mathrm{j} \omega)$ and $U_{j}(\mathrm{j} \omega)$ are the Fourier transforms of $y_{i}$ and $u_{j}$ respectively. These FRFs are determined (non-parametrically) by applying swept sine waves, in the frequency band of $5-200 \mathrm{~Hz}$, to the piezoelectric actuators (including the central patch corresponding to the disturbance term $w$ ) and measuring the corresponding output signals $y_{t i p}, v_{p 1}$ and $v_{p 2}$. The inputs and the outputs, with exception to $y_{t i p}$ were generated and measured respectively using an HP36570A dual channel spectrum analyser. The tip displacement $y_{\text {tip }}$, was measured using a Polytec laser scanning vibrometer (PSV-300). In Figure 8, the nonparametric FRFs $G_{i j}(\mathrm{j} \omega), i, j=1,2$ and 3 are plotted. It is apparent from the plots that all the FRFs have three resonance frequencies in the plotted frequency band, and not surprisingly the resonance frequencies are the same for all the FRFs. A model of the form

$$
\begin{aligned}
\ddot{X}+M \dot{X}+\Omega X & =\Psi u(t)+\beta w(t) \\
V_{p}(t) & =\Psi^{\top} X+D_{v u} u(t)+D_{v w} w(t) \\
y_{t i p}(t) & =P^{\top} X+D_{y_{t i p} u} u(t)+D_{y_{t i p} w} w(t)
\end{aligned}
$$

was fit for the data plotted in Figure 8. It can be observed from Figure 8 that the model fits the data reasonably well. As there are three modes $X$ is a $3 \times 1$ vector, $M$ and $\Omega$ are $3 \times 3$ diagonal matrix, $\Psi, D_{v u}$ and $D_{v w}$ are $3 \times 2,2 \times 2$ and $2 \times 1$ matrices respectively, $\beta$ and $P$ are $3 \times 1$ vectors, and $D_{y_{t i p} u}$ and $D_{y_{t i p} \frac{w}{w}}$ are scalars. Here, $u(t) \triangleq\left[v_{1}, v_{2}\right]^{\top}$ and $V_{p}(t) \triangleq\left[v_{p 1}, v_{p 2}\right]^{\top}$. The details of the estimation of the model (24), though by no means trivial, are not crucial for the paper and hence omitted.

\section{B. Controller Design}

The tip displacement $y_{t i p}$ of the beam gives a measure of the vibrations in the beam. As $w$ represents a disturbance, the FRF $G_{11}(\mathrm{j} \omega) \triangleq G_{y_{t i p} w}(\mathrm{j} \omega)=Y_{t i p}(\mathrm{j} \omega) / W(\mathrm{j} \omega)$, where $Y_{\text {tip }}(\mathrm{j} \omega)$ and $W(\mathrm{j} \omega)$ are the Fourier transforms of $y_{t i p}(t)$ and $w(t)$ respectively, is a good indicator of the effect of noise on the beam. A well damped FRF $G_{y_{t i p} w}(\mathrm{j} \omega)$ would imply a well damped system. Hence, here, a controller $K(s)$, (12), is designed such that the closed loop FRF $G_{C l, y w}(\mathrm{j} \omega)$ corresponding to the input $w$ and output $y_{\text {tip }}$ is well damped. 

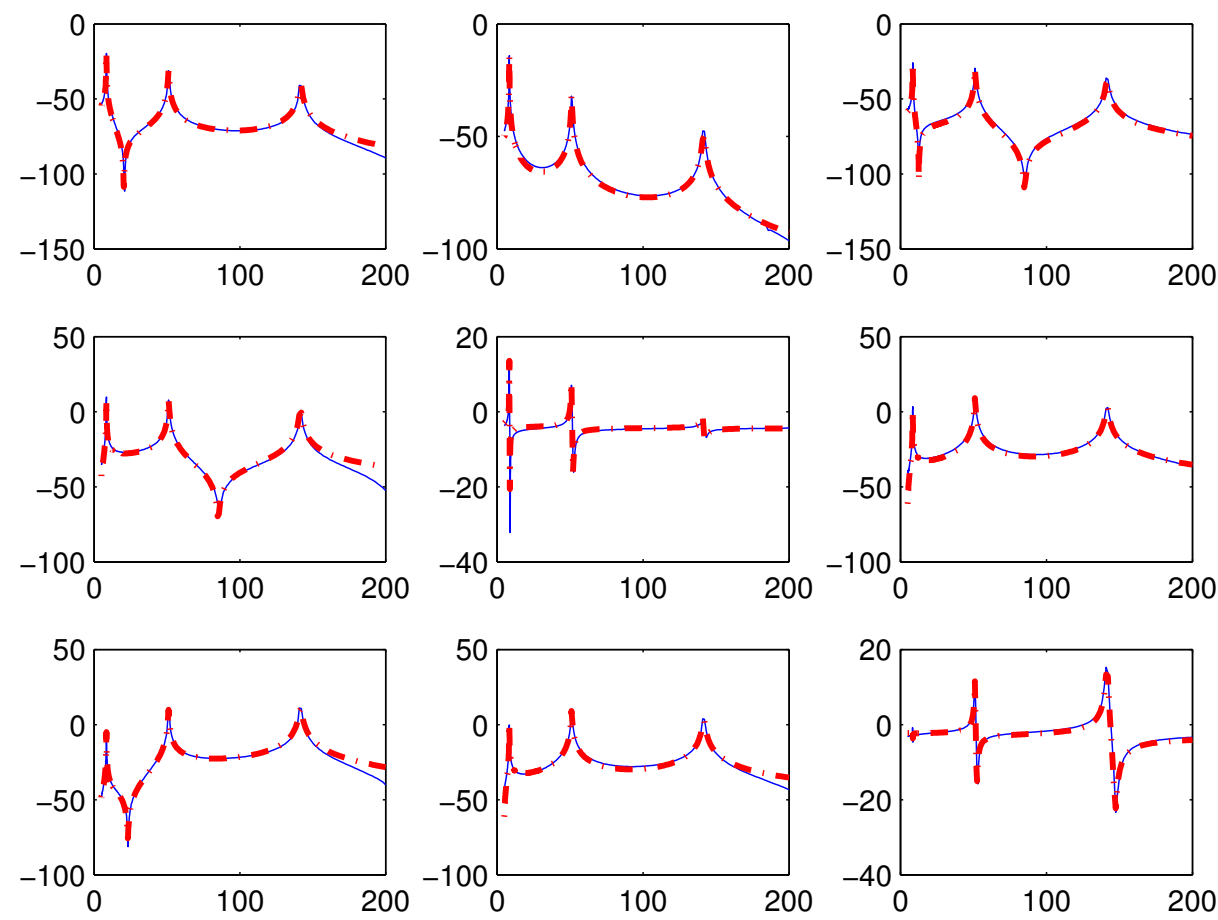

Fig. 8. Identified model (-.) with measured data (-)

Alternatively stated, a controller is designed such that the poles of the closed loop FRF $G_{C l, y w}(\mathrm{j} \omega)$ are well inside the left half plane.

Here, for technical ease (24) is rewritten in the standard state space from

$$
\begin{aligned}
\dot{x}(t) & =A x(t)+B_{v} u(t)+B_{w} w(t) \\
V_{p}(t) & =C_{v} x(t)+D_{v u} u(t)++D_{v w} w(t) \\
y_{t i p}(t) & =C_{y_{t i p}} x(t)+D_{y_{t i p} u} u(t)+D_{y_{t i p} w} w(t),
\end{aligned}
$$

where

$$
\begin{aligned}
& A=\left[\begin{array}{ccccc}
0 & 1 & & 0 & 0 \\
-\omega_{1}^{2} & -2 \zeta_{1} \omega_{1} & & 0 & 0 \\
& & \ddots & & \\
0 & 0 & & 0 & 1 \\
0 & 0 & & -\omega_{3}^{2} & -2 \zeta_{3} \omega_{3}
\end{array}\right] \\
& B=\left[\begin{array}{lll}
B_{w} & B_{v_{1}} & B_{v_{2}}
\end{array}\right]=\left[\begin{array}{ccc}
0 & 0 & 0 \\
\beta_{1} & \Psi_{1}^{v_{1}} & \Psi_{1}^{v_{2}} \\
\vdots & \vdots & \vdots \\
0 & 0 & 0 \\
\beta_{3} & \Psi_{3}^{v_{1}} & \Psi_{3}^{v_{2}}
\end{array}\right] \\
& C=\left[\begin{array}{c}
C_{y_{t i p}} \\
C_{v_{1}} \\
C v_{2}
\end{array}\right]=\left[\begin{array}{ccccc}
P_{1} & 0 & \ldots & P_{3} & 0 \\
\Psi_{1}^{v_{1}} & 0 & \ldots & \Psi_{3}^{v_{1}} & 0 \\
\Psi_{1}^{v_{2}} & 0 & \ldots & \Psi_{3}^{v_{2}} & 0
\end{array}\right],
\end{aligned}
$$

where $\left\{\omega_{k}^{2}\right\}_{k=1}^{3}$ and $\left\{2 \zeta_{k} \omega_{k}\right\}_{k=1}^{3}$ are the diagonal elements of the matrices $\Omega$ and $M$ respectively, $\{\beta\}_{k=1}^{3}$ are the elements of the $3 \times 1$ vector $\beta,\left\{\Psi_{k}^{v_{1}}\right\}_{k=1}^{3}$ and $\left\{\Psi_{k}^{v_{2}}\right\}_{k=1}^{3}$ are elements of the first and the second columns of $\Psi$ matrix and $\left\{P_{k}\right\}_{k=1}^{3}$ are the elements of the $P$ vector. As $K(s)$ acts as a feedback controller connecting the plant output $V_{p}$ to the input $u$, the control dynamics, in time domain, is governed by

$$
\begin{aligned}
\tilde{x} & =\Gamma D \tilde{x}+\Gamma V_{p} \\
u & =\tilde{x} .
\end{aligned}
$$

Setting $Z=[x, \tilde{x}]$ the closedloop system can be written as

$$
\begin{aligned}
\dot{Z} & =\left[\begin{array}{cc}
A & B_{u} \\
\Gamma C_{v} & \Gamma\left(D+D_{v u}\right)
\end{array}\right] Z+\left[\begin{array}{c}
B_{w} \\
\Gamma D_{v w}
\end{array}\right] w \\
y_{t i p} & =\left[C_{t i p}, D_{y_{t i p} u}\right] Z+D_{y_{t i p} w} w(t) .
\end{aligned}
$$

The goal is to choose $\Gamma$ and $D$ such the poles of (28) are well into to the left half plane. To this end the following optimization problem is posed,

$$
\min _{\Gamma, D} \sum_{k=1}^{10}\left|P_{k}^{(d)}-P_{k}^{(c)}(\Gamma, D)\right|
$$

under the constraints, $\Gamma>0$ and $-D>G(0)=\Psi \Omega^{-1} \Psi^{\top}$. In (29) $P_{k}^{(d)}$, s denote the set of desired closed loop poles, while $P_{k}^{(c)}(\Gamma, D)$ are the closed loop pole positions for a given $\Gamma$ and $D$. In an ideal scenario one would like the have $\Gamma$ and $D$ such that $P_{k}^{(d)}=P_{k}^{(c)}(\Gamma, D)$ for all $k$. Here, the desired closed loop poles are set to $\left\{P_{k}^{(d)}=\right.$ $\left.p_{k}-15\right\}_{k=1}^{6}$, where $\left\{p_{k}\right\}_{k=1}^{6}$ are the open loop poles and $P_{7}^{(d)}=P_{8}^{(d)}=-30$. The cost-function is minimised using 


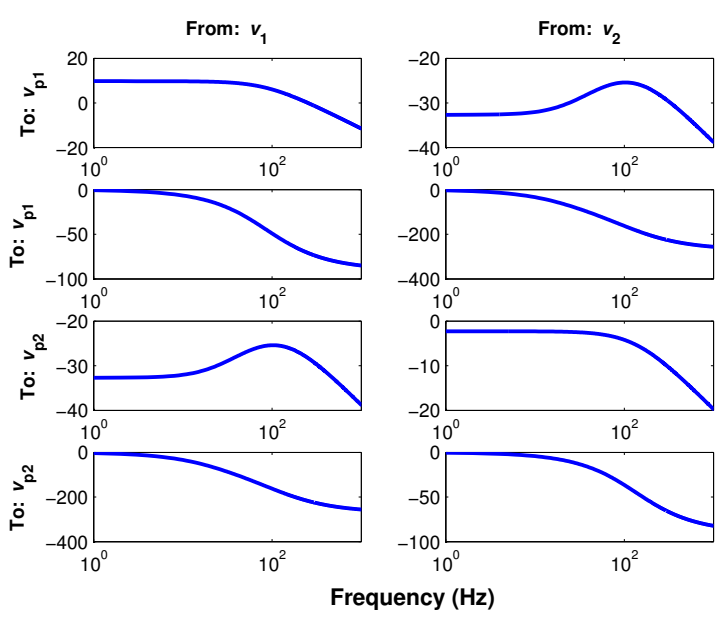

Fig. 9. Magnitude plot of the controller $K(s)$

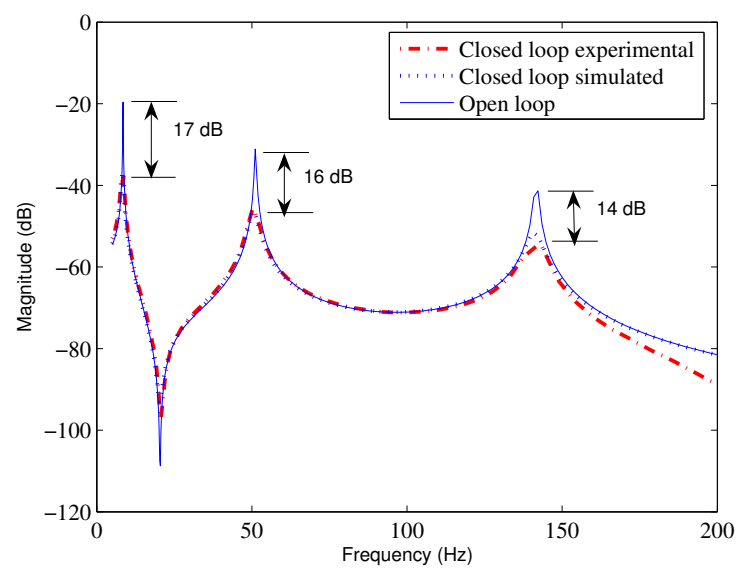

Fig. 10. Magnitude plot of the closed loop transfer function, $G_{C l, y_{t i p} w}$ both simulated and experimentally determined along with the open loop.

a non-linear search with the constraints enforced. Needless to say, the cost function (29) is non-convex and has many local minimas. Therefore, the controller obtained here is only locally optimal. In Figure 9 the magnitude plot of the controller obtained for the desired closed loop poles $P_{k}^{(d)}, k=$ $1,2, \ldots, 6$ is presented. The effect of this controller is tested both numerically (Simulated) and experimentally. It can be observed from Figure 10 that the numerically predicted (simulated) closed loop transfer-function matches reasonably with the experimentally determined one. Furthermore a good damping of all the three resonances can be seen from the plots.

\section{CONCLUDING DISCUSSION}

In this paper an extension of the integral resonant control (IRC) technique to resonant MIMO systems with collocated sensor-actuator pairs was considered. The extension of the IRC from the SISO systems (with one collocated sensor- actuator pair) to the MIMO systems (with several collocated sensor-actuator pairs), though a natural progression, was by no means a straight-forward one. While the closed loop stability in the SISO case was investigated using rootlocus criterion, the proof in MIMO case necessitated the use of more advanced and recent mathematical machinery. It was shown that for collocated resonant structures IRC controller guarantees closed loop stability subject to certain LMI constraints. Thereby making the class of such stabilizing controllers a convex set. A constrained optimization procedure was proposed for designing multivariable IRCs which can deliver sufficient damping. A multivarible IRC was designed for and implemented on an active structure with two pairs of collocated piezoelectric sensor/actuator pairs. The mulivariable IRC controllers were shown to add significant damping to the active structure.

The procedure presented in this paper for designing IRC controllers involves minimizing a nonlinear nonconvex cost function over a convex set. The nonconvex nature of the cost function makes the control design heavily reliant on the initial guess or the starting point of the nonlinear search used for minimizing the cost function. A convex optimization approach to IRC design is still an open question.

\section{REFERENCES}

[1] B. Bushan, Ed., Springer Hand book of Nanotechnology, Heidelberg, Germany, Springer-Verlag 2004.

[2] S. Wu, T. L. Turner, S. A. Rizzi, "Piezoelectric shunt vibration damping of an F-16 panel under high acoustic excitation", Smart Structures and Materials: Damping and Isolation, Proc of SPIE 3989, 276-287.

[3] M. J. Balas, "Feedback control of flexible systems", IEEE Transactions on Automatic Control, vol AC-23, no 4, pp 673-679, Aug 1978.

[4] C. W. de Silva, Vibration Fundamentals and Practice, CRC Press, 1999.

[5] J. Juang and M. Q. Phan, Identification and Control of Mechanical Systems, Cambridge University Press, 2001.

[6] S. O. R. Moheimani and B. J. G. Vautier, "Resonant Control of Vibration Using Charge-Driven Piezoelectric Actuators", IEEE Transactions on Control Systems Technology, vol 13, no 6, pp 1021-1035, 2005.

[7] J. L. Fanson and T. K. Caughey, "Positive position feedback control for large space structures", AIAA JOURNAL, vol 28, no 4, pp 717-724, 1990.

[8] S. O. R. Moheimani, B. J. G. Vautier, B. Bhikkaji, IEEE Transactions on Control Systems Technology, vol 14, no 3, pp 443-445, 2006.

[9] S. S. Aphale, A. J. Fleming and S. O. R. Moheimani, "Integral control of smart structures", Smart Materials and Structures, vol 16, 2007, pp 439-446.

[10] A. Lanzon, I. R. Petersen, "Stability robustness of a feedback interconnection of systems with negative imaginary frequency response", IEEE Transactions on Automatic Control, vol 53, 2008, pp 1042-1046.

[11] S. O. R. Moheimani and D. Halim and A. J. Fleming, Spatial Control of Vibration: Theory and Experiments, World Scientific, 2002.

[12] K. Zhou, J. C. Doyle and K. Glover, Robust and Optimal Control, Prentice Hall, New Jersey, USA, 1995.

[13] A. J. Laub,Matrix Analysis for Scientists and Engineers,SIAM, Philadelphia, USA, 2005.

[14] D. S. Bernstein, Matrix Mathematics, Princeton University Press, New Jersy, USA, 2005. 